\title{
Phagocytosis and activation of bone marrow-derived macrophages by Plasmodium falciparum gametocytes
}

Yolanda Corbett ${ }^{1,6^{*}} \mathbb{0}$, Silvia Parapini ${ }^{2,6}$, Federica Perego ${ }^{3}$, Valeria Messina ${ }^{4}$, Serena Delbue ${ }^{3}$, Paola Misiano ${ }^{1}$, Mario Falchi ${ }^{5}$, Francesco Silvestrini ${ }^{4,6}$, Donatella Taramelli ${ }^{1,6}$, Nicoletta Basilico ${ }^{3,6}$ and Sarah D'Alessandro $3,6,7^{*}$

\begin{abstract}
Background: The innate immune response against various life cycle stages of the malaria parasite plays an important role in protection against the disease and regulation of its severity. Phagocytosis of asexual erythrocytic stages is well documented, but little and contrasting results are available about phagocytic clearance of sexual stages, the gametocytes, which are responsible for the transmission of the parasites from humans to mosquitoes. Similarly, activation of host macrophages by gametocytes has not yet been carefully addressed.

Methods: Phagocytosis of early or late Plasmodium falciparum gametocytes was evaluated through methanol fixed cytospin preparations of immortalized mouse C57BI/6 bone marrow-derived macrophages treated for $2 \mathrm{~h}$ with $P$. falciparum and stained with Giemsa, and it was confirmed through a standardized bioluminescent method using the transgenic P. falciparum 3D7elo1-pfs16-CBG99 strain. Activation was evaluated by measuring nitric oxide or cytokine levels in the supernatants of immortalized mouse C57BI/6 bone marrow-derived macrophages treated with early or late gametocytes.

Results: The results showed that murine bone marrow-derived macrophages can phagocytose both early and late gametocytes, but only the latter were able to induce the production of inflammatory mediators, specifically nitric oxide and the cytokines tumour necrosis factor and macrophage inflammatory protein 2.

Conclusions: These results support the hypothesis that developing gametocytes interact in different ways with innate immune cells of the host. Moreover, the present study proposes that early and late gametocytes act differently as targets for innate immune responses.
\end{abstract}

Keywords: Malaria, Plasmodium falciparum gametocytes, Immortalized mouse C57BI/6 bone marrow-derived macrophages, Phagocytosis, Nitric oxide, Tumour necrosis factor-alpha, Macrophage inflammatory protein 2

\section{Background}

Malaria is a vector-borne parasitic disease caused by the protozoan Plasmodium and transmitted by female

\footnotetext{
*Correspondence: ycorbett179@gmail.com; sarah.dalessandro@unimi.it ${ }^{1}$ Dipartimento di Scienze Farmacologiche e Biomolecolari, Università degli Studi di Milano, via Pascal 36, 20133 Milan, Italy

${ }^{7}$ Present Address: Dipartimento di Scienze Farmacologiche e

Biomolecolari, Università degli Studi di Milano, via Pascal 36, 20133 Milan, Italy

Full list of author information is available at the end of the article
}

Anopheles mosquitoes. In 2019, 229 million cases of malaria and 409,000 deaths have been reported, mostly due to Plasmodium falciparum and Plasmodium vivax, two of the five species which infect humans [1].

The life cycle of Plasmodium involves two hosts, the mosquito vector and a vertebrate. In humans, parasites develop in the liver without symptoms for the host, whereas the symptomatic phase starts with the asexual replication inside red blood cells. After rounds of asexual intraerythrocytic replication, a small proportion of

(c) The Author(s) 2021. This article is licensed under a Creative Commons Attribution 4.0 International License, which permits use, sharing, adaptation, distribution and reproduction in any medium or format, as long as you give appropriate credit to the original author(s) and the source, provide a link to the Creative Commons licence, and indicate if changes were made. The images or other third party material in this article are included in the article's Creative Commons licence, unless indicated otherwise in a credit line to the material. If material is not included in the article's Creative Commons licence and your intended use is not permitted by statutory regulation or exceeds the permitted use, you will need to obtain permission directly from the copyright holder. To view a copy of this licence, visit http://creativeco mmons.org/licenses/by/4.0/. The Creative Commons Public Domain Dedication waiver (http://creativecommons.org/publicdomain/ zero/1.0/) applies to the data made available in this article, unless otherwise stated in a credit line to the data. 
parasites develop into sexual forms called gametocytes (GCT). Male and female GCT undergo five stages of differentiation, from I to V [2]. Stage V GCT circulate for several days until they are taken up by the vector, where the sporogonic sexual cycle occurs leading to the production of sporozoites, which can be injected into a new host. GCT have been found in the bone marrow (BM), spleen, gut, and brain of post-mortem samples from malaria patients, although the presence in BM is much higher and reported in different studies [3, 4]. GCT are also present in the BM of malaria patients [5, 6], including anaemic children [7].

Differently from $P$. falciparum asexual blood stages, which sequester in the deep vasculature via specific endothelial receptors [8], GCT lack specific adhesion molecules, do not adhere to the endothelium nor to primary erythroblasts, and have been found in the BM extravascular space $[3,5,9-12]$. The presence of Plasmodium GCT in the BM suggests that this microenvironment may sustain both infection and transmission [13-15]. However, at present, the exact molecular mechanism of GCT sequestration in the BM is not clear. The BM niche where GCT reside is characterized by the presence of many cell types, both haematopoietic and non-haematopoietic. Among these, macrophages play a crucial role in haematopoietic stem cells retention and survival and erythroid maturation [16]. One of the hypotheses concerning the presence of gametocytes in the BM is that they develop inside erythroblasts, thus affecting erythropoiesis [17]. In the $\mathrm{BM}$ of malaria patients, mature asexual parasites and haemozoin, the malarial pigment, are also found [18].

There is a gap of knowledge on the role of macrophages in the development and survival of GCT as well as on the effect of GCT on the activation and functions of BM macrophages.

Macrophages are the major effector of innate immunity. In malaria, splenic macrophages are responsible for the direct and antibody-dependent elimination of infected red blood cells (RBC) [19, 20]. Monocytes/ macrophages from different organs are also activated by parasite antigens or products to release cytokines and chemokines and toxic mediators [21]. However, most of the data in the literature describe the response of macrophages to asexual blood-stage parasites. Contrasting data are available on the relationship between GCT and macrophages, on GCT phagocytosis, and macrophage activation by GCT [22-25].

The present work aimed to investigate some macrophage responses induced by GCT in BM, the place where GCT develop. In particular, the interactions between BM macrophages and early or late GCT were studied through the examination of phagocytosis of GCT and the induction of pro-inflammatory cytokines or nitric oxide (NO).

\section{Methods \\ Reagents}

Unless otherwise stated, reagents for cell and parasite culture were from EuroClone S.p.A (Pero, Italy), whereas all other reagents were from Sigma Aldrich S.r.l. (Milano, Italy).

\section{Cell culture}

Immortalized mouse $\mathrm{C} 57 \mathrm{Bl} / 6 \mathrm{BM}$-derived macrophages (BMDM) from wild type lineage were kindly provided by Drs. Douglas Golenbock and Kate Fitzgerald, UMASS (MA, USA) [26, 27]. Mycoplasma-free BMDM were maintained in Dulbecco's minimal essential medium (DMEM) complemented with $2 \mathrm{mM}$ L-glutamine, $20 \mathrm{mM}$ HEPES, and $10 \%$ heat-inactivated FBS, as previously reported. The cultures were maintained at $37{ }^{\circ} \mathrm{C}$ in an atmosphere with $5 \% \mathrm{CO}_{2}$.

\section{Parasites culture}

The transgenic P. falciparum 3D7elo1-pfs16-CBG99 strain expressing the Pyrophorus plagiophthalamus CBG99 luciferase under the gametocyte specific promoter pfs16 was used in all the experiments $[28,29]$. The strain was kindly provided by Dr Pietro Alano at ISS (Rome, Italy). Mycoplasma-free P. falciparum parasites were maintained in vitro at $5 \%$ haematocrit (human type 0-positive) RBC in RPMI-1640 supplemented with $10 \%(\mathrm{v} / \mathrm{v})$ naturally-clotted heat-inactivated (human type 0-positive) (Interstate Blood Bank, Inc. [USA]) serum, $0.37 \mathrm{mM}$ hypoxanthine, $2 \mathrm{mM} \mathrm{L}$-glutamine, and $25 \mathrm{mM}$ HEPES. The cultures were maintained at $37{ }^{\circ} \mathrm{C}$ in a standard gas mixture consisting of $1 \% \mathrm{O}_{2}, 5 \% \mathrm{CO}_{2}$, and $94 \%$ $\mathrm{N}_{2}$ [30].

GCT were obtained as previously described [30]. Briefly, asexual parasites were diluted with fresh RBC and the medium was changed daily without further addition of RBC, to obtain a parasitaemia higher than $5 \%$. The cultures were treated with $50 \mathrm{mM} \mathrm{N}$-acetylglucosamine (NAG) to clear residual asexual parasites and to obtain virtually pure GCT cultures. Early GCT (stage I-III GCT) were obtained after 4-5 days of NAG treatment, while late GCT (>95\% stage V) after 12-14 days. The GCT stages were identified and counted through Giemsa-stained smears.

\section{GCT purification}

GCT were purified by magnetic separation using MACS columns (Miltenyi ${ }^{\circledR}$ ), based on the paramagnetic properties of haemozoin, which is present in GCT. Cultures at $\sim 0.5 \%$ haematocrit were placed onto an LS column 
to retain and remove the free haemozoin, as described earlier [31]. The LS column elution, which contains GCT, was transferred onto an LD column, where GCT remained attached. Finally, GCT were eluted from this column with fresh medium and then centrifuged. Both total RBC and GCT were counted at an optical microscope in a Neubauer cell counting chamber. The percentage of parasitaemia was confirmed in Giemsa-stained smears.

\section{Incubation of immortalized mouse C57BI/6 bone marrow-derived macrophages with GCT}

BMDM were plated in 96-well plates at a concentration of $10^{5}$ cells/well in $100 \mu \mathrm{l} /$ well of cell medium and left adhere overnight. Experiments for luminescence reading were performed in 96-well white plates, whereas experiments for $\mathrm{NO} /$ cytokines/chemokines production in transparent plates. Only for evaluation of NO production, cells were pre-treated with interferon-gamma $($ IFN- $\gamma)(100 \mathrm{U} / \mathrm{ml})$ for $2 \mathrm{~h}$. BMDM were stimulated with medium, late ( $>90 \%$ stage V) or early GCT (stage I-III) for $2 \mathrm{~h}$ in shaking conditions. In some experiments, cells were pre-treated for $1 \mathrm{~h}$ with $2 \mu \mathrm{M}$ cytochalasin $\mathrm{D}$ to inhibit phagocytosis. GCT were added to cells in $100 \mu \mathrm{l} /$ well volume in the GCT medium. The cell:GCT ratio was 1:1.5-2.

Uninfected RBC at the same haematocrit were used as controls in all the experiments. Lipopolysaccharide (LPS) $(100 \mathrm{ng} / \mathrm{ml})$ was used as a positive control for $\mathrm{NO} /$ cytokine/chemokines production.

\section{Luminescent determination of GCT}

GCT and BMDM were co-incubated for different time points, the supernatants were removed and cells were washed with PBS. To completely remove not-phagocytized GCT, cells were treated with ice-cold distilled water for $20 \mathrm{~s}$, a treatment which does not affect cell viability, but osmotically lyses RBC, as described by Schwarzer and colleagues [32]. BMDM were washed with PBS to remove remaining traces of haemoglobin and to restore cellular physiologic conditions. Next, $70 \mu \mathrm{l}$ of PBS and $70 \mu \mathrm{l}$ of D-luciferin ( $1 \mathrm{mM}$ in citrate buffer $0.1 \mathrm{M}, \mathrm{pH} 5.5$ ) were added to each well and incubated for 20-30 min in the dark. Absolute Luminescent Units (ALU) were read with a microplate reader Synergy4 $\left(\right.$ Biotek $\left.^{\circledR}\right)$, using an integration time of $500 \mathrm{~ms}$.

\section{Microscopic observation}

BMDM were seeded in 24-well plates $\left(2.5 \times 10^{5} /\right.$ well in $600 \mu \mathrm{l}$ ), incubated with GCT, and then detached by trypsinization. Cells were centrifuged and cytospin was performed to spot cells onto slides. Alternatively, cells were plated and treated with GCT in 16-well chamber slides previously treated with poly-L-lysine MW70000$150000(0.1 \mathrm{mg} / \mathrm{ml})$ to increase cell adhesion $\left(\right.$ Labtek $\left.^{\circledR}\right)$. Slides were fixed in absolute methanol and stained with a $0.4 \%$ Giemsa solution (w/v) prepared in buffered methanol solution with stabilizers and diluted in a $\mathrm{pH} 6.5$ buffer. Images were taken with an inverted microscope (Nikon Ti-S) at $1000 \times$ magnification (immersion oil 100x objective) and the digital camera Nikon Digital Sight.

For confocal microscopy, BMDM were plated in 8-well or 16-well chamber slides previously treated with polyL-lysine $(0.1 \mathrm{mg} / \mathrm{ml})$ and left adhere overnight. GCT were pre-treated for $30 \mathrm{~min}$ with $3 \mu \mathrm{M}$ of a fluorescent marker (CellTracker ${ }^{\mathrm{TM}}$ RedCMTPX Dye, Molecular Probes), then washed and incubated with BMDM for $2 \mathrm{~h}$ in shaking conditions. At the end of incubation, cells were washed with PBS and fixed with paraformaldehyde (formalin solution $4 \%$ [Sigma]) for 15 min. After further PBS washing, cell membranes were stained with $10 \mu \mathrm{g} / \mathrm{ml}$ WGAFITC (Wheat germ agglutinin, DBA Italia). Smears were mounted using the Fluoroshield ${ }^{\mathrm{TM}}$ mounting medium containing DAPI for nuclei staining. Images were taken on an inverted microscope equipped with a confocal spectral imaging system (Olympus Fluoview 1000, Tokyo, Japan) at $600 \times$ magnification.

\section{Determination of nitric oxide production by immortalized mouse C57BI/6 bone marrow-derived macrophages}

Accumulation of nitrite in cell supernatants was evaluated by Griess assay [33, 34] as an indirect measure of NO production, as described in different models reported by Corbett and colleagues [31]. The Griess reagent is a $50 \%$ $\mathrm{v} / \mathrm{v}$ mixture of Reagent A ( $1 \% \mathrm{w} / \mathrm{v}$ sulphanilamide) and Reagent B (0.1\% w/v naphthyl ethylenediamine dihydrochloride and $2.5 \% \mathrm{w} / \mathrm{v}$ phosphoric acid). One hundred microlitres of Griess reagent were added to $100 \mu \mathrm{l}$ of sample supernatants. After $10 \mathrm{~min}$ incubation at room temperature, the absorbance was read at $540 \mathrm{~nm}$, using a Synergy 4 microplate reader (Biotek, GE). A standard curve of sodium nitrite $\left(\mathrm{NaNO}_{2}\right)$ was used to extrapolate the nitrite levels of samples.

\section{Determination of cytokine/chemokine production by immortalized mouse C57BI/6 bone marrow-derived macrophages}

The production of tumour necrosis factor (TNF) and macrophage inflammatory protein 2 (MIP-2) was evaluated using commercially available ELISA kits (R\&D DuoSet) following the manufacturer's instructions.

\section{Statistical analysis}

Data were analysed by one-way or two-way ANOVA followed by posthoc multiple comparisons tests (Dunnett's, 
Tukey's), using the software GraphPad Prism 6, as reported in figure legends.

\section{Results}

Evaluation of GCT phagocytosis using a luminescent-based bioassay

The bioassay to quantify GCT phagocytosis by BMDM was set up using luminescent late GCT ( $>95 \%$ stage V) from the transgenic $P$. falciparum 3D7elo1-pfs16-CBG99 strain cultured with BMDM in shaking conditions. The phagocytic activity of BMDM was confirmed by using latex beads. After $2 \mathrm{~h}$ of co-incubation, cells were washed to remove non-phagocytized parasites. To optimize the assay and eliminate the luminescent signal of non-phagocytized GCT, a lysis step with water was introduced before the addition of luciferin. The luminescent signal associated with macrophages was taken as a measure of GCT phagocytosis. As shown in Fig. 1a, a significant reduction in the luminescent signal was observed in the group exposed to lysis with water indicating that the lysis step is required to avoid unspecific signal and thus overestimation of phagocytosis. The water lysis step has been performed in all the subsequent experiments.

To confirm that the luminescence signal was produced by the phagocytosed GCT, the second set of experiments was performed pre-incubating BMDM with Cytochalasin $\mathrm{D}$, an inhibitor of actin polymerization and thus of cell phagocytosis [35]. As shown in Fig. 1b, in the presence of Cytochalasin D, the luminescent signal disappeared, indicating that the method is suitable to study phagocytosis.

Time course experiments were performed to assess gametocytes viability and luminescence after phagocytosis over time. Results indicated that luminescence of GCT inside BMDM increased from 0 to $30-60 \mathrm{~min}$ and remained stable up to $150 \mathrm{~min}$, suggesting that GTC are still luminescent at $2 \mathrm{~h}$ after phagocytosis (Fig. 1c).

Phagocytosis of GCT was visualized and confirmed by different microscopic techniques. Figure 2a shows representative pictures of Giemsa-stained smears of BMDM containing late GCT in a vacuole in the cytoplasm after $2 \mathrm{~h}$ of co-culture. These data were confirmed by confocal microscopy analysis, where GCT, cell membranes, and nuclei were stained with different fluorescent markers to localize intracellular GCT. The presence of red GCT inside macrophages, stained green for membranes and blue for nuclei, was confirmed (Fig. 2b, c).

\section{Phagocytosis of early and late GCT}

The luciferase assay was utilized to evaluate the phagocytosis of late (>90\% stage V) versus early (stage I-III) GCT. BMDM were incubated with late or early GCT for $2 \mathrm{~h}$, at the cell:GCT ratio of 1:1.5. The luminescent

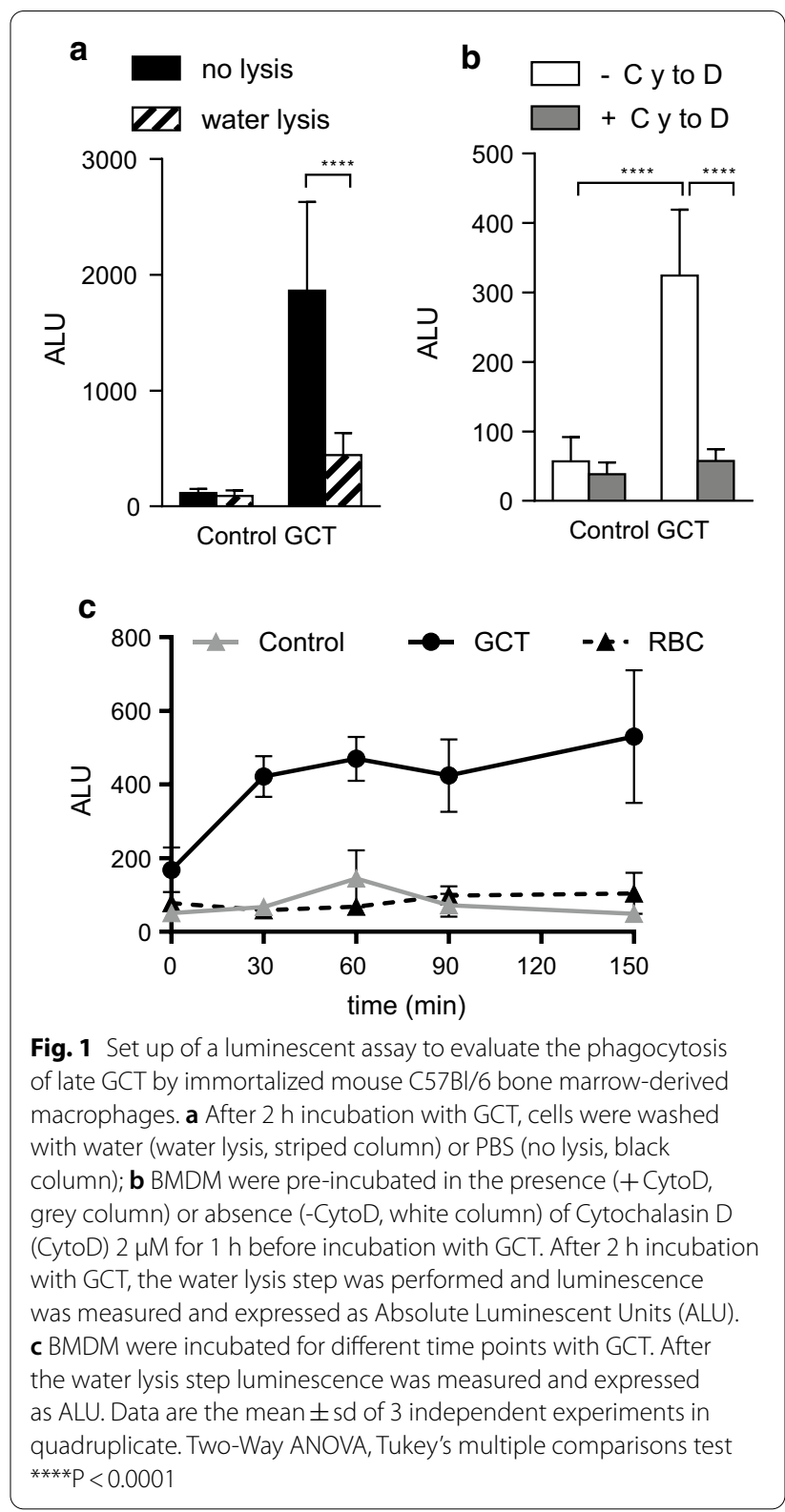

signal was proportional to the number of GCT used in the assay. However, the luminescence was higher in the groups treated with late GCT compared to early GCT (Fig. 3a), suggesting greater phagocytosis of late GCT than early GCT.

To confirm the previous observations and be able to quantify the number of phagocytized GCT, the luciferase activity of serially diluted early or late GCT was evaluated. This was particularly crucial since it has been reported that the luciferase accumulates in 3D7elo1-pfs16-CBG99 GCT during their development, and thus the luminescent signal is higher in late versus early GCT [29]. 

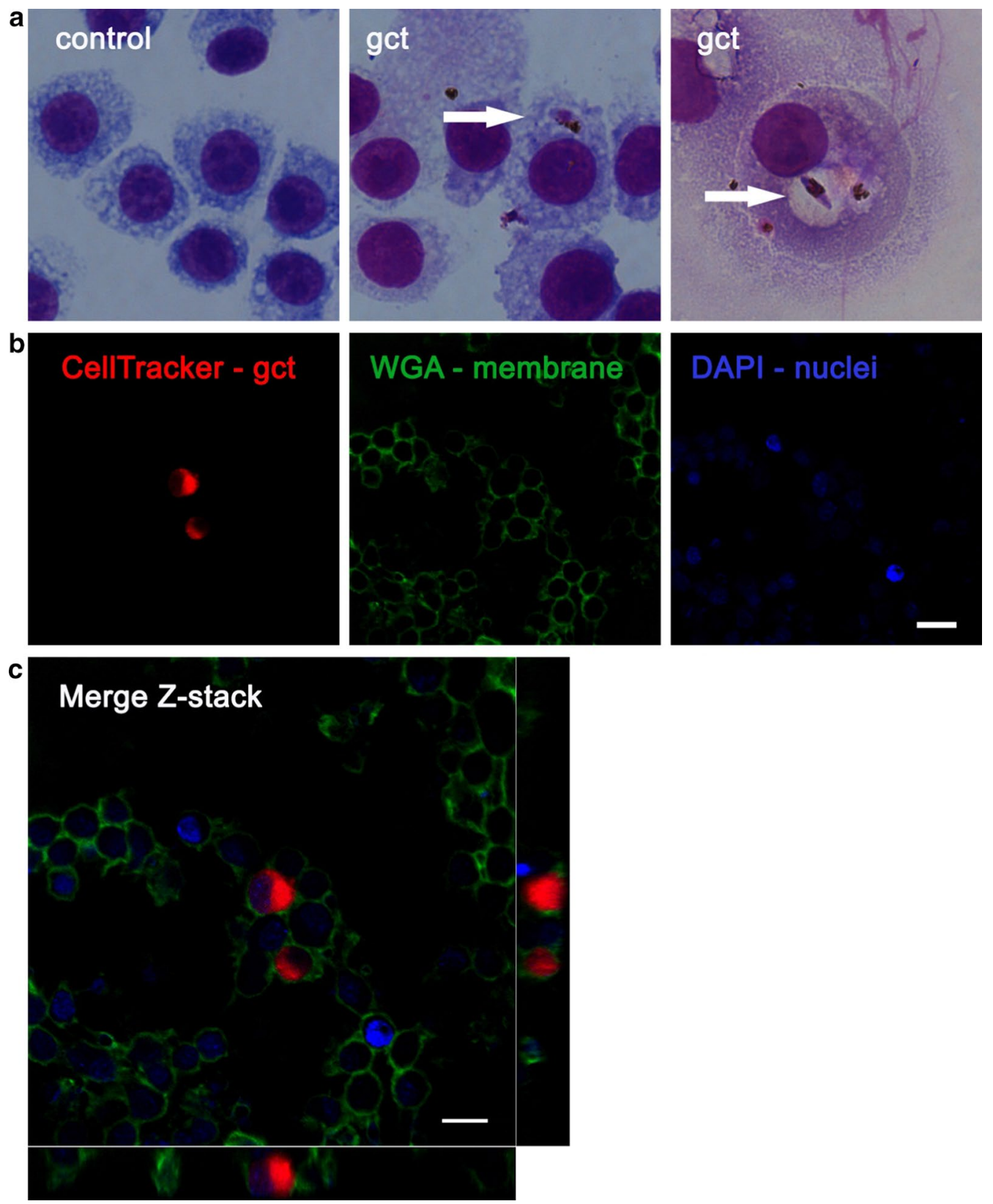

Fig. 2 Microscopic observation of phagocytosis of late GCT by immortalized mouse C57BI/6 bone marrow-derived macrophages. a BMDM were incubated with GCT for $2 \mathrm{~h}$, detached from the plate, spotted on smears by cytospin centrifugation, fixed in absolute methanol, and stained with Giemsa. The arrows indicate GCT inside macrophages. Pictures, from one representative experiment out of three, were taken at $\times 1000$ magnification. b GCT were incubated with $3 \mu \mathrm{M}$ CellTracker ${ }^{\mathrm{TM}}$ RedCMTPX Dye for $30 \mathrm{~min}$ to stain cytoplasm (red in the picture). BMDM were incubated for $2 \mathrm{~h}$ with GCT and fixed with formalin $4 \%$. BMDM membranes were stained with $10 \mu \mathrm{g} / \mathrm{ml}$ WGA-FITC (Wheat germ agglutinin, DBA Italia) (green in the picture). BMDM nuclei were stained by the DAPI present in the mounting medium (Fluoroshield) (blue in the picture). $\mathbf{c} Z$-stack images were taken on an inverted microscope equipped with a confocal spectral imaging system (Olympus Fluoview 1000, Tokyo, Japan) 600x magnification. Scale bar, $10 \mu \mathrm{m}$

As reported in Fig. 3b, a linear correlation between the number of GCT and the mean ALU values was observed. Late GCT mean ALU values were approximately $20-40 \%$ higher than that of early GCT. The difference between early and late GCT and the linearity of the luciferase activity was maintained also at low GCT number and low ALU, as shown in Fig. 3c. These curves were used to extrapolate the number of GCT 
a

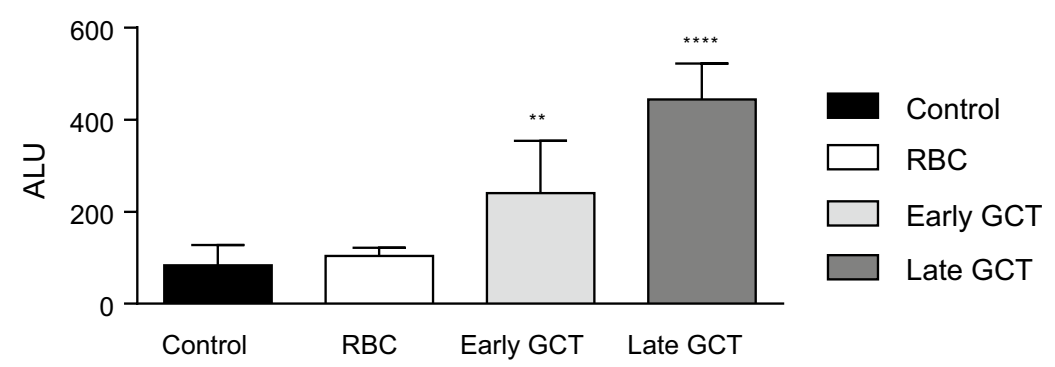

b

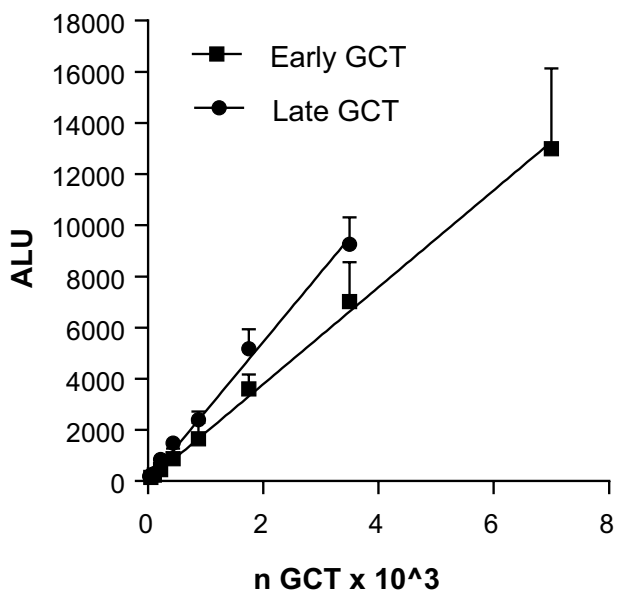

C

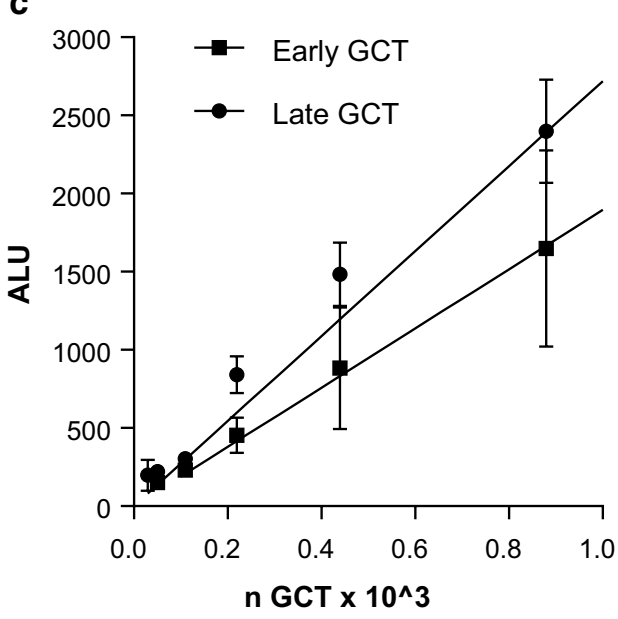

\begin{tabular}{|l|c|c|}
\hline BMDM treatment & ALU & GCT number \\
\hline Control & $94 \pm 22$ & 0 \\
\hline Early GCT & $258 \pm 62$ & $70 \pm 33$ \\
\hline Late GCT & $539 \pm 170$ & $82 \pm 23$ \\
\hline
\end{tabular}

d

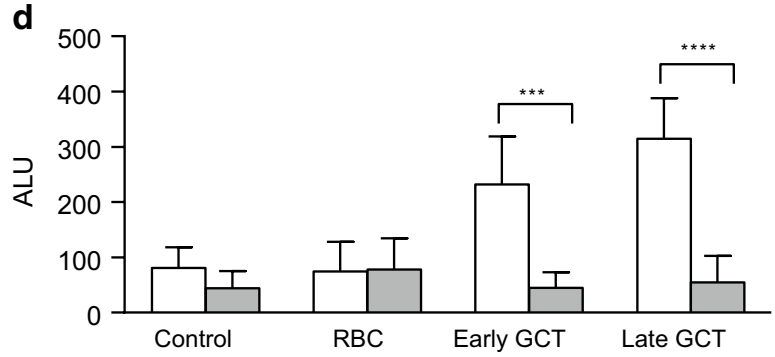

Fig. 3 Immortalized mouse C57BI/6 bone marrow-derived macrophages were treated with early or late GCT at the cell:GCT ratio of 1:1.5, for $2 \mathrm{~h}$. a After washing and lysis, luminescence was read using a Biotek Synergy4 microplate reader. Results are expressed as arbitrary luminescence units (ALU). ${ }^{*} \mathrm{p}<0.05$; ${ }^{* * * *} \mathrm{P}<0.0001$ versus control, One-Way ANOVA, Dunnett's multiple comparison test. $\mathbf{b}$ Linear correlation between the number of GCT and the relative luminescence in two different dose-response curves, one for late GCT, one for early GCT. c Magnification of the lower part of b. The table shows the luminescence emitted (ALU) by the medium alone, or by early or late GCT (BMDM treatment), and the numbers of GCT phagocytized by BMDM extrapolated from the dose-response curve shown in c (GCT number). $\mathbf{d}$ BMDM were pre-incubated in the presence (+ CytoD, grey column) or absence (-CytoD, white column) of Cytochalasin D (CytoD) $2 \mu \mathrm{M}$ for $1 \mathrm{~h}$ before incubation with GCT. After $2 \mathrm{~h}$ incubation with GCT, the water lysis step was performed and luminescence was measured and expressed as Absolute Luminescent Units (ALU). ${ }^{* *} p<0.001$; ****P $<0.0001$ versus control, Two-Way ANOVA, Tuckey's multiple comparison test. Data are the mean \pm sd of 3 independent experiments in triplicate 
phagocytized by BMDM. The number of early and late GCT phagocytized by BMDM was $70 \pm 33$ and $82 \pm 23$, respectively. It appears that, when corrected for the luciferase activity of each GCT population, the extent of phagocytosis of early vs. late GCT was not significantly different.

The same experiments were performed pre-incubating BMDM with Cytochalasin D and, as expected, in the presence of Cytochalasin $\mathrm{D}$ the luminescent signal from BMDM incubated with gametocytes was significantly reduced and was comparable to controls (Fig. 3d).

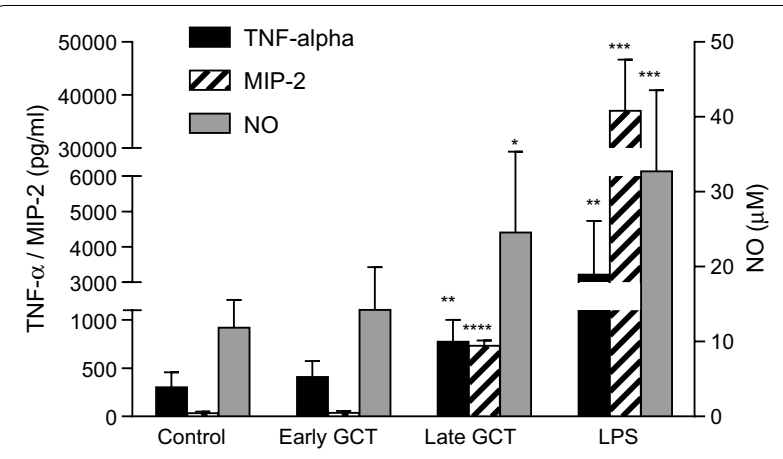

Fig. 4 Immortalized mouse C57BI/6 bone marrow-derived macrophages were treated with early or late GCT at the cell:GCT ratio of 1:2, for $24 \mathrm{~h}$. The production of TNF and MIP-2 was evaluated in the supernatants by ELISA. Nitric oxide production was evaluated by measuring nitrite in cell supernatants by Griess assay. LPS $(100 \mathrm{ng} / \mathrm{ml})$ was used as a positive control. Data are the mean \pm SD of 3 independent experiments in triplicate. ${ }^{*} p<0.05 ;{ }^{* *} p<0.01$; ${ }^{* * *} p<0.001 ;{ }^{* * *} \mathrm{P}<0.0001$ versus control, Two-Way ANOVA, Dunnett's multiple comparison test
Production of nitric oxide and cytokines by immortalized mouse C57BI/6 bone marrow-derived macrophages treated with GCT

The ability of GCT to activate BMDM was evaluated by measuring the production of inflammatory mediators in the culture supernatants after $24 \mathrm{~h}$. As shown in Fig. 4, late GCT induced a 2-fold increase in the production of both TNF and NO, compared to unstimulated controls. The CXC chemokine MIP-2 was also strongly induced. On the contrary, the stimulation of BMDM by early GCT was not significantly different from untreated controls. LPS $(100 \mathrm{ng} / \mathrm{ml})$ was used as a positive stimulus.

To address whether NO and cytokine production was due to phagocytosis or to contact with gametocytes, experiments were repeated in the presence of cytochalasin D. Results show that cytochalasin D did not modify the production of $\mathrm{NO}$ and increased the production of both TNF and MIP-2 induced by late GCT (Fig. 5) proving that phagocytosis was not involved in cytokines and $\mathrm{NO}$ induction. On the contrary, the introduction of a water lysis step abrogated the production of $\mathrm{NO}$ and cytokines induced by GCT indicating that $\mathrm{NO}$ and cytokines production was due to the contact of GCT and cells and not to the phagocytized parasites.

\section{Discussion}

Gametocytes (GCT) have a key role in malaria, being the sexual blood stage of the parasite responsible for transmission. There is controversial information in the literature about the human immune responses elicited by GCT. The present study demonstrated that BMDM phagocytized both early and late P. falciparum GCT, but only late GCT induced the production of inflammatory mediators.
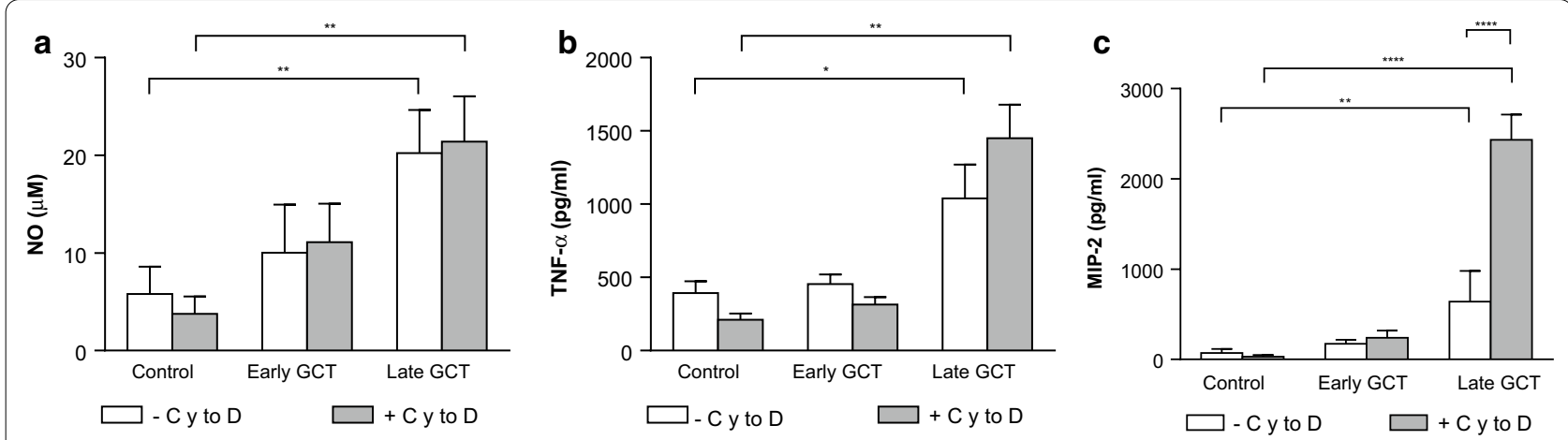

Fig. 5 Immortalized mouse C57BI/6 bone marrow-derived macrophages were pre-incubated in the presence ( + CytoD, grey column) or absence (-CytoD, white column) of Cytochalasin D (CytoD) $2 \mu \mathrm{M}$ for $1 \mathrm{~h}$ before incubation with early or late GCT at the cell:GCT ratio of 1:2, for $24 \mathrm{~h}$. The production of NO was evaluated by measuring nitrite in cell supernatants by Griess assay. The production of TNF and MIP-2 was evaluated in the supernatants by ELISA. Data are the mean \pm sd of 3 independent experiments in triplicate. ${ }^{*} p<0.05 ;{ }^{* *} p<0.01 ;{ }^{* * *} p<0.001 ;{ }^{* * *} p<0.0001$ versus control, Two-Way ANOVA, Tukey's multiple comparison test 
The phagocytosis of GCT by macrophages was observed by light microscopy on Giemsa-stained cytospin preparations and confirmed by confocal analysis, using different fluorescent probes to label and identify BMDM or GCT structures. The latter technique allowed the visualization of cells in 3 dimensions, which unequivocally determined the presence of GCT inside the cells. Moreover, the present work describes a simple bioluminescent method able to measure the presence of GCT inside BMDM. The transgenic 3D7elo1-pfs16CBG99 P. falciparum strain expresses the potent luciferase CBG99 under the control of the GCT specific promoter Pfs16 and produces luminescence only in the GCT stage after the addition of luciferin [28]. The luminescence associated with BMDM after treatment with GCT was used as a measure of phagocytosis. To increase the sensitivity and specificity of the assay, the cells were carefully washed after phagocytosis of GCT, and lysis of non-phagocytized GCT was performed, preserving BMDM viability. Using an inhibitor of phagocytosis [35], the luminescent signal disappeared, confirming the reliability of the method. Data reporting phagocytosis of $P$. falciparum GCT in the literature are few and controversial, probably due to differences in the cellular models, isolation protocols, and GCT stage used to perform the experiments. Late GCT are not phagocytized by human differentiated THP-1 monocytes [23], whereas they are phagocytized by peripheral blood mononuclear cells, even if less efficiently compared to asexual stages or gametes [24]. Stage I-II GCT, but not stage IV GCT, are phagocytized by macrophages derived by human blood or murine peritoneal cavity with a CD36- dependent mechanism [22]. Up to now, phagocytosis of GCT by macrophages has been demonstrated mostly by using Giemsa staining and optical microscopy, a time-consuming method, highly dependent on operator skills. Moreover, optical microscopy does not allow discriminating precisely between parasites which are inside cells from parasites which are attached on the surface. All these limitations were overcome by using confocal microscopy and by the luminescent method proposed in this work.

The luminescent method is simple and reliable and was applied to compare the extent of phagocytosis of early versus late GCT. In this respect, the luminescence emitted by different numbers of early versus late GCT confirmed that the luminescent signal was higher in late compared to early GCT, as previously reported [29], but the extent of phagocytosis was similar. However, the efficiency of phagocytosis of both late and early stages was low, in line with the results of Healer and colleagues [24], which showed that phagocytosis of GCT was limited when compared to that of asexual parasites or gametes.
The method can be easily applied to other in vitro models using transgenic luminescent microorganisms.

The observation here that GCT were phagocytized by BMDM reaffirms that innate immunity plays an important role in malaria. Several studies show that whole asexual $P$. falciparum-infected erythrocytes or their parasite products induce macrophages activation $[21,26,36]$. In particular, the malaria pigment, haemozoin, which is also present in GCT, is a potent pro-inflammatory stimulus for macrophages, leading to the activation of the toll-like receptors, inflammasome complex, and NOD2 pathway $[26,36,37]$.

Anyhow, immune reactions may also involve sexual stages, affecting malaria transmission [25, 38-41]. Although the involvement of specific immunity mediated by antibodies against GCT and gametes antigens [42] was described [43], the role of innate immunity has not been well examined so far. Data from the literature show that there is a detrimental activity of cytokines or NO on GCT infectivity $[38,44]$.

The data presented here showed that late but not early GCT induced TNF, a pleiotropic cytokine with a range of activities [45, 46], including endothelial activation leading to the sequestration of asexual parasitized $R B C$ in cerebral vessels during the severe forms of malaria [47, 48]. As stated before, several data from the literature reveal the absence of specific adhesion molecules on GCT. Rogers and colleagues showed that TNF increases the adhesion of early GCT to the endothelium of the BM [11]. Induction of TNF by late GCT described in the present manuscript suggested the potential role of this cytokine in the homing of early GCT to the BM, where they develop into late stages [3,9-11].

In the same manner, only late GCT induced macrophage inflammatory protein MIP-2, a major CXC chemokine (CXCL2) involved in the recruitment of polymorphonuclear neutrophils to the sites of inflammation (reviewed in [49]). Previous studies from the authors of the present work showed that MIP-2 is upregulated in BMDM stimulated by malaria pigment [37]. This is the first evidence of the involvement of MIP-2 in GCT-macrophage interaction in BM. Matzer et al. [50] showed that MIP-2 is constitutively expressed in $\mathrm{BM}$, but not in other organs like the spleen, lung, or liver. Interestingly, MIP-2 has a role in leukocyte adhesion in the BM [51]. Thus, MIP-2 induction by late GCT is worth further investigation.

Again, only late GCT induced NO production from BMDM. NO is a potent vasodilator and cellular mediator involved in many biologic functions, which participates in the pathogenesis of severe malaria and in particular of cerebral malaria [52]. NO is important not only in the human host but also in the vector since it contributes to the immune responses of mosquitoes against 
Plasmodium infection [53]. Moreover, NO increases parasite deformability, thus inducing the stage V GCT release from the BM niche towards circulation [54]. The results presented here sustain this hypothesis.

Even in the absence of GCT internalization, BMDM produced inflammatory mediators in response to GCT. Indeed, when cytochalasin D was used to prevent GCT phagocytosis, the production of NO was comparable in the presence and absence of cytochalasin $\mathrm{D}$. The production of TNF and MIP-2 induced by GCT was even increased in the presence of cytochalasin D indicating that phagocytosis was not needed for BMDM stimulation. An increase of cytokines production by cells treated with cytochalasin $\mathrm{D}$ have been already described although the effect of cytochalasin D can be both stimulating and inhibiting depending on macrophages origin, type of pathogen and experimental model [55-57].

\section{Conclusions}

The present work showed that early and late GCT are phagocytized by immortalized macrophages from BM cells but only late GCT induce the production of inflammatory mediators (i.e. TNF, MIP-2, or NO), regardless of phagocytosis. Thus, the data available in the literature, already discussed above, and the results presented here, suggest that innate immune responses elicited by late GCT, at least in BM cells, may have a dual role. On one side, they may establish a hostile milieu, inducing late GCT to escape from the bone marrow towards circulation. On the other side, they may support the retention and thus the development of early GCT in the bone marrow.

\section{Abbreviations}

ALU: Absolute luminescent units; DMEM: Dulbecco's minimal essential medium; early GCT: Stage I-III GCT; GCT: Gametocytes; BMDM: Immortalized mouse $\mathrm{C} 57 \mathrm{BI} / 6$ bone marrow (BM)-derived macrophages; IFN- $\gamma$ : Interferongamma; late GCT: Stage V; LPS: Lipopolysaccharide; MIP-2: Macrophage inflammatory protein 2; NAG: N-Acetylglucosamine; NO: Nitric oxide; RBC: Red blood cells; TNF: Tumour necrosis factor; WGA: Wheat germ agglutinin.

\section{Acknowledgements}

Authors thank Dr Pietro Alano for kindly providing the P. falciparum strain; Drs. Golenbock and Fitzgerald for immortalized BMDM. Drs Laura Galastri, Paola Verducci, and Tiziana Bianchi from AVIS Comunale Milano are gratefully acknowledged for providing blood samples for parasite culture.

\section{Authors' contributions}

YC, SP, FP, VM, MF, FS, SD'A performed experiments; YC, FS, NB, SD'A, SD analysed results; $Y C, N B$, SD'A designed the research; $Y$ C, FS, SD'A, PM made the figures. YC, SD'A, PM, FS, DT, NB, SD wrote the paper. All the authors contributed equally to the revision of the final manuscript.

\section{Funding}

This work was supported by "Fondazione Cariplo" (grant number 2017 - 0846), by Ministero dell'Istruzione, dell'Università e della Ricerca (PRIN 2015.4JRJPP_004) and by the Ministero degli Affari Esteri e della Cooperazione Internazionale (Progetti di grande rilevanza 00949-2018).
Availability of data and materials

Not applicable.

Ethics approval and consent to participate

Not applicable.

Consent for publication

Not applicable.

\section{Competing interests}

The authors declare no competing interests.

\section{Author details}

${ }^{1}$ Dipartimento di Scienze Farmacologiche e Biomolecolari, Università degli Studi di Milano, via Pascal 36, 20133 Milan, Italy. ${ }^{2}$ Dipartimento di Scienze Biomediche per la Salute, Università degli Studi di Milano, Milan, Italy. ${ }^{3}$ Dipartimento di Scienze Biomediche, Chirurgiche e Odontoiatriche, Università degli Studi di Milano, via Pascal 36, 20133 Milan, Italy. ${ }^{4}$ Dipartimento di Malattie Infettive, Istituto Superiore di Sanità, Rome, Italy. ${ }^{5}$ AIDS-Ricerca e sviluppo, Istituto Superiore di Sanità, Rome, Italy. ${ }^{6}$ Centro Interuniversitario di Ricerca sulla Malaria-Italian Malaria Network, Milan, Italy. ${ }^{7}$ Present Address: Dipartimento di Scienze Farmacologiche e Biomolecolari, Università degli Studi di Milano, via Pascal 36, 20133 Milan, Italy.

Received: 20 December 2019 Accepted: 8 January 2021

Published online: 10 February 2021

\section{References}

1. WHO. World Malaria Report. Geneva, World Health Organization, 2020.

2. Ngotho P, Soares AB, Hentzschel F, Achcar F, Bertuccini L, Marti M. Revisiting gametocyte biology in malaria parasites. FEMS Microbiol Rev. 2019;43:401-14.

3. Joice R, Nilsson SK, Montgomery J, Dankwa S, Egan E, Morahan B, et al. Plasmodium falciparum transmission stages accumulate in the human bone marrow. Sci Transl Med. 2014;6:244re245.

4. De Niz M, Meibalan E, Mejia P, Ma S, Brancucci NMB, Agop-Nersesian C, et al. gametocytes display homing and vascular transmigration in the host bone marrow. Sci Adv. 2018:4:eaat3775.

5. Farfour E, Charlotte F, Settegrana C, Miyara M, Buffet P. The extravascular compartment of the bone marrow: a niche for Plasmodium falciparum gametocyte maturation? Malar J. 2012;11:285.

6. Abdulsalam AH, Sabeeh N, Bain BJ. Immature Plasmodium falciparum gametocytes in bone marrow. Am J Hematol. 2010;85:943.

7. Aguilar R, Magallon-Tejada A, Achtman AH, Moraleda C, Joice R, Cisteró $P$, et al. Molecular evidence for the localization of Plasmodium falciparum immature gametocytes in bone marrow. Blood. 2014;123:959-66.

8. Craig AG, Khairul MF, Patil PR. Cytoadherence and severe malaria. Malays $J$ Med Sci. 2012:19:5-18.

9. Silvestrini F, Tibúrcio M, Bertuccini L, Alano P. Differential adhesive properties of sequestered asexual and sexual stages of Plasmodium falciparum on human endothelial cells are tissue independent. PLoS One. 2012;7:e31567.

10. Rogers NJ, Targett GA, Hall BS. Plasmodium falciparum gametocyte adhesion to C32 cells via CD36 is inhibited by antibodies to modified band 3 . Infect Immun. 1996;64:4261-8.

11. Rogers NJ, Hall BS, Obiero J, Targett GA, Sutherland CJ. A model for sequestration of the transmission stages of Plasmodium falciparum: adhesion of gametocyte-infected erythrocytes to human bone marrow cells. Infect Immun. 2000:68:3455-62.

12. Neveu G, Dupuy F, Ladli M, Barbieri D, Naissant B, Richard C, et al. Plasmodium falciparum gametocyte-infected erythrocytes do not adhere to human primary erythroblasts. Sci Rep. 2018;8:17886.

13. Nilsson SK, Childs LM, Buckee C, Marti M. Targeting human transmission biology for malaria elimination. PLoS Pathog. 2015;11:e1004871.

14. Alano P. The emerging role of the human bone marrow as a privileged developmental niche for the transmission stages of the malaria parasite Plasmodium falciparum. Ann Ist Super Sanita. 2017;53:96-9.

15. Messina V, Valtieri M, Rubio M, Falchi M, Mancini F, Mayor A, et al. Gametocytes of the malaria parasite Plasmodium falciparum interact with and 
stimulate bone marrow mesenchymal cells to secrete angiogenetic factors. Front Cell Infect Microbiol. 2018;8:50.

16. Birbrair A, Frenette PS. Niche heterogeneity in the bone marrow. Ann NY Acad Sci. 2016;1370:82-96.

17. Neveu G, Richard C, Dupuy F, Behera P, Volpe F, Subramani PA, et al. Plasmodium falciparum sexual parasites develop in human erythroblasts and affect erythropoiesis. Blood. 2020;136:1381-93.

18. Smalley ME, Abdalla S, Brown J. The distribution of Plasmodium falciparum in the peripheral blood and bone marrow of Gambian children. Trans R Soc Trop Med Hyg. 1981;75:103-5.

19. Del Portillo HA, Ferrer M, Brugat T, Martin-Jaular L, Langhorne J, Lacerda MV. The role of the spleen in malaria. Cell Microbiol. 2012;14:343-55.

20. Buffet PA, Safeukui I, Deplaine G, Brousse V, Prendki V, Thellier M, et al. The pathogenesis of Plasmodium falciparum malaria in humans: insights from splenic physiology. Blood. 2011;117:381-92.

21. Ortega-Pajares A, Rogerson SJ. The rough guide to monocytes in malaria infection. Front Immunol. 2018;9:2888.

22. Smith TG, Serghides L, Patel SN, Febbraio M, Silverstein RL, Kain KC. CD36-mediated nonopsonic phagocytosis of erythrocytes infected with stage I and IIA gametocytes of Plasmodium falciparum. Infect Immun. 2003;71:393-400

23. Bansal GP, Weinstein CS, Kumar N. Insight into phagocytosis of mature sexual (gametocyte) stages of Plasmodium falciparum using a human monocyte cell line. Acta Trop. 2016;157:96-101.

24. Healer J, Graszynski A, Riley E. Phagocytosis does not play a major role in naturally acquired transmission-blocking immunity to Plasmodium falciparum malaria. Infect Immun. 1999;67:2334-9.

25. Dantzler KW, Ma S, Ngotho P, Stone WJR, Tao D, Rijpma S, et al. Naturally acquired immunity against immature. Sci Transl Med. 2019;11:eaav3963.

26. Kalantari P, Deoliveira RB, Chan J, Corbett Y, Rathinam V, Stutz A, et al. Dual engagement of the NLRP3 and AIM2 inflammasomes by Plasmodiumderived hemozoin and DNA during malaria. Cell Rep. 2014;6:196-210.

27. Hornung V, Bauernfeind F, Halle A, Samstad EO, Kono H, Rock KL, et al. Silica crystals and aluminum salts activate the NALP3 inflammasome through phagosomal destabilization. Nat Immunol. 2008;9:847-56.

28. Cevenini L, Camarda G, Michelini E, Siciliano G, Calabretta MM, Bona $\mathrm{R}$, et al. Multicolor bioluminescence boosts malaria research: quantitative dual-color assay and single-cell imaging in Plasmodium falciparum parasites. Anal Chem. 2014;86:8814-21.

29. D'Alessandro S, Camarda G, Corbett Y, Siciliano G, Parapini S, Cevenini $L$, et al. A chemical susceptibility profile of the Plasmodium falciparum transmission stages by complementary cell-based gametocyte assays. $J$ Antimicrob Chemother. 2016;71:1148-58.

30. D'Alessandro S, Silvestrini F, Dechering K, Corbett Y, Parapini S, Timmerman $\mathrm{M}$, et al. A Plasmodium falciparum screening assay for anti-gametocyte drugs based on parasite lactate dehydrogenase detection. J Antimicrob Chemother. 2013:68:2048-58.

31. Corbett $Y$, D'Alessandro S, Parapini S, Scaccabarozzi D, Kalantari P, Zava $\mathrm{S}$, et al. Interplay between Plasmodium falciparum haemozoin and L-arginine: implication for nitric oxide production. Malar J. 2018;17:456.

32. Schwarzer E, Turrini F, Ulliers D, Giribaldi G, Ginsburg H, Arese P. Impairment of macrophage functions after ingestion of Plasmodium falciparum-infected erythrocytes or isolated malarial pigment. J Exp Med. 1992;176:1033-41.

33. Prato M, D'Alessandro S, Van den Steen PE, Opdenakker G, Arese P, Taramelli $\mathrm{D}$, et al. Natural haemozoin modulates matrix metalloproteinases and induces morphological changes in human microvascular endothelium. Cell Microbiol. 2011;13:1275-85.

34. Griess J. Bemerkungen zu der abhandlung der H.H. Weselsky und Benedikt "Ueber einige azoverbindungen.". Chemische Berichte. 1879;12:426-8.

35. Elliott JA, Winn WC. Treatment of alveolar macrophages with cytochalasin D inhibits uptake and subsequent growth of Legionella pneumophila. Infect Immun. 1986:51:31-6.

36. Parroche P, Lauw FN, Goutagny N, Latz E, Monks BG, Visintin A, et al. Malaria hemozoin is immunologically inert but radically enhances innate responses by presenting malaria DNA to Toll-like receptor 9. Proc Natl Acad Sci USA. 2007:104:1919-24.

37. Corbett Y, Parapini S, D'Alessandro S, Scaccabarozzi D, Rocha BC, Egan TJ, et al. Involvement of Nod 2 in the innate immune response elicited by malarial pigment hemozoin. Microbes Infect. 2015;17:184-94.
38. Naotunne TS, Karunaweera ND, Mendis KN, Carter R. Cytokine-mediated inactivation of malarial gametocytes is dependent on the presence of white blood cells and involves reactive nitrogen intermediates. Immunology. 1993;78:555-62.

39. Long GH, Chan BH, Allen JE, Read AF, Graham AL. Blockade of TNF receptor 1 reduces disease severity but increases parasite transmission during Plasmodium chabaudi chabaudi infection. Int J Parasitol. 2008;38:1073-81.

40. Naotunne TS, Karunaweera ND, Del Giudice G, Kularatne MU, Grau GE, Carter R, et al. Cytokines kill malaria parasites during infection crisis: extracellular complementary factors are essential. J Exp Med. 1991;173:523-9.

41. Stone WJ, Dantzler KW, Nilsson SK, Drakeley CJ, Marti M, Bousema T, et al. Naturally acquired immunity to sexual stage $P$. falciparum parasites. Parasitology. 2016;143:187-98.

42. Vermeulen AN, Ponnudurai T, Beckers PJ, Verhave J, Smith MA, Meuwissen $\mathrm{JH}$. Sequential expression of antigens on sexual stages of Plasmodium falciparum accessible to transmission-blocking antibodies in the mosquito. J Exp Med. 1985;162:1460-76.

43. Contreras CE, Ploton IN, Siliciano RF, Karp CL, Viscidi R, Kumar N. Mapping of specific and promiscuous HLA-DR-restricted T-cell epitopes on the Plasmodium falciparum 27-kilodalton sexual stage-specific antigen. Infect Immun. 1998;66:3579-90.

44. Ascenzi P, Gradoni L. Nitric oxide limits parasite development in vectors and in invertebrate intermediate hosts. IUBMB Life. 2002;53:121-3.

45. Chakravorty SJ, Carret C, Nash GB, Ivens A, SzestakT, Craig AG. Altered phenotype and gene transcription in endothelial cells, induced by Plasmodium falciparum-infected red blood cells: pathogenic or protective? Int J Parasitol. 2007:37:975-87.

46. Treeratanapiboon L, Psathaki K, Wegener J, Looareesuwan S, Galla HJ, Udomsangpetch $\mathrm{R}$. In vitro study of malaria parasite induced disruption of blood-brain barrier. Biochem Biophys Res Commun. 2005;35:810-8.

47. Dunst J, Kamena F, Matuschewski K. Cytokines and chemokines in cerebral malaria pathogenesis. Front Cell Infect Microbiol. 2017;7:324.

48. Turner GD, Morrison H, Jones M, Davis TM, Looareesuwan S, Buley ID, et al. An immunohistochemical study of the pathology of fatal malaria. Evidence for widespread endothelial activation and a potential role for intercellular adhesion molecule-1 in cerebral sequestration. Am J Pathol. 1994;145:1057-69.

49. Kobayashi Y. The role of chemokines in neutrophil biology. Front Biosci. 2008;13:2400-7.

50. Matzer SP, Baumann T, Lukacs NW, Röllinghoff M, Beuscher HU. Constitutive expression of macrophage-inflammatory protein 2 (MIP-2) mRNA in bone marrow gives rise to peripheral neutrophils with preformed MIP-2 protein. J Immunol. 2001;167:4635-43.

51. Burdon PC, Martin C, Rankin SM. The CXC chemokine MIP-2 stimulates neutrophil mobilization from the rat bone marrow in a CD49d-dependent manner. Blood. 2005;105:2543-8.

52. Percário S, Moreira DR, Gomes BA, Ferreira ME, Gonçalves AC, Laurindo PS, et al. Oxidative stress in malaria. Int J Mol Sci. 2012;13:16346-72.

53. Tahar R, Boudin C, Thiery I, Bourgouin C. Immune response of Anopheles gambiae to the early sporogonic stages of the human malaria parasite Plasmodium falciparum. EMBO J. 2002;21:6673-80.

54. Ramdani G, Naissant B, Thompson E, Breil F, Lorthiois A, Dupuy F, et al. CAMP-signalling regulates gametocyte-infected erythrocyte deformability required for malaria parasite transmission. PLoS Pathog. 2015;11:e1004815.

55. Patel NR, Bole M, Chen C, Hardin CC, Kho AT, Mih J, et al. Cell elasticity determines macrophage function. PLoS One. 2012;7:e41024.

56. Dietrich N, Rohde M, Geffers R, Kröger A, Hauser H, Weiss S, et al. Mast cells elicit proinflammatory but not type I interferon responses upon activation of TLRs by bacteria. Proc Natl Acad Sci USA. 2010;107:8748-53.

57. Kim MY, Kim JH, Cho JY. Cytochalasin B modulates macrophage-mediated inflammatory responses. Biomol Ther (Seoul). 2014;22:295-300.

\section{Publisher's note}

Springer Nature remains neutral with regard to jurisdictional claims in published maps and institutional affiliations. 